\title{
Exploration of underlying molecular mechanism of Lycii Cortex in Treating Type 2 Diabetes Mellitus Based on Network Pharmacology and Molecular Docking
}

\author{
Dongjun $\mathrm{Li}^{1}$, Denghui Wang ${ }^{1}$, Shikai Yan ${ }^{1,2^{*}}$ \\ 1. School of Traditional Chinese Medicine, Guangdong Pharmaceutical University, Guangzhou 510006, China \\ 2. School of Pharmacy, Shanghai Jiao Tong University, Shanghai 200240, China
}

\begin{abstract}
Objective: To explore the potential molecular mechanism of Lycii Cortex in treating type 2 diabetes mellitus (T2DM) by virtue of network pharmacology and molecular docking method. Methods: Ingredients of Lycii Cortex were collected from TCMSP and BATMAN-TCM databases, and the corresponding targets and T2DM-related targets were obtained respectively from SwissTargetPrediction and GenCards databases. Venn diagram was applied to derive the potential active components and effect targets of Lycii Cortex in the treatment of T2DM. GO enrichment analysis and KEGG pathway analysis were performed in the database of DAVID. Cytoscape 3.6.1 was used to produce the "core components-core targets" network. The molecular docking between core components and core targets was implemented through Autodock Vina. Results: Six core components and twelve core targets of Lycii Cortex in treating T2DM were identified. GO enrichment analysis and KEGG pathway analysis suggested the following signaling pathways and biological processes were involved in the treatment of T2DM by Lycii Cortex: PI3K-Akt signaling pathway, TNF signaling pathway, HIF-1 signaling pathway, Toll-like receptor signaling pathway, NOD-like receptor signaling pathway, and peptidyl-threonine phosphorylation, the positive regulation of cyclase activity, the positive regulation of genetic expression, and lipoprotein translocation. The binding results demonstrated a relatively high affinity between core components of Lycii Cortex, including kulactone, hederagenin, scopolin, etc., and core targets, containing IL6, PPAR $\gamma$, TNF, and mTOR, indicating the efficacy of Lycii Cortex in treating T2DM. Conclusion: Lycii Cortex plays a role in the treatment of T2DM with its ingredients such as kulactone, linarin, hederagenin, and scopolin regulating glycometabolism, affecting cell apoptosis and weakening inflammatory response through targets like IL6, PPAR $\gamma$, TNF, and mTOR.
\end{abstract}

\section{INTRODUCTION}

Diabetic Mellitus (DM) serves as one type of metabolic disease directly triggered by genetic factors and coinduced by a series of external factors such as environment and dietary habit ${ }^{[1]}$. It represents a syndrome primarily featured by disturbed glucose, protein, and fat metabolism with glycometabolism disturbance as the leading symptom, which is caused by insulin decline and blood glucose increase owing to impaired insulin secretion, bringing a serious threat to human health ${ }^{[2]}$. According to the latest report released by International Diabetes Federation, 116.4 million adult DM patients are living in China now and this figure is expected to climb to 147.2 million by 2045 , of which $95 \%$ are T2DM patients ${ }^{[3]}$. At present, the existing common treatments include the injection of insulin and oral administration of drugs, which have delivered outstanding effects. Nevertheless, the longterm application of these methods tends to develop multiple complications, different degrees of drug resistance, as well as insulin resistance. ${ }^{[4,5,6]}$ Over recent years, traditional Chinese medicines have emerged as a crucial role in the treatment of T2DM since both the single application of traditional Chinese medicines or its combined application with western medicines can effectively control the level of blood glucose, significantly improve clinical symptoms, and largely prevent the appearance of diabetic complication ${ }^{[7]}$.

Lycii Cortex, the dried root bark of Lycium Chinense Mill. or Lycium barbarum L., is equipped with the functions of cooling the blood, clearing away bonesteaming heat and lung heat, and lowering the evil fire and has been clinically applied to treat symptoms like yin deficiency, tidal fever, bone-steaming fever, night sweats, lung heat, cough, intrinsic heat, and wasting-thirst ${ }^{[8]}$. Pharmacological researches have exhibited its antiviral and antibacterial activity with the ability to reduce the level of blood pressure and blood glucose and regulate blood lipid ${ }^{[9,10]}$. Although many studies have reported the treatment of T2DM by Lycii Cortex ${ }^{[11,12,13]}$, the mechanism of action still requires in-depth investigation. For this reason, this paper is designed to preliminarily explore the underlying molecular mechanism of Lycii Cortex in 
treating T2DM on the basis of network pharmacology and molecular docking technology.

By taking system biology as its foundation and integrating different technologies such as polypharmacology and network analysis, network pharmacology can systematically uncover the connection between effect targets of drugs and disease targets through constructing different layers of networks ${ }^{[14,15]}$. Molecular docking is aimed at predicting and simulating the binding mode and affinity between small molecular compositions of medicines and target proteins of disease by matching their shape and energy ${ }^{[16]}$. By now, the combined application of network pharmacology and molecular docking technology has emerged as a productive tool in investigating the material basis and mechanism of traditional Chinese medicine and compound medicine in disease treatment ${ }^{[17]}$. On this basis, this study is designed to predict the potential active components, effect targets, and mechanism of Lycii Cortex in treating T2DM by adopting network pharmacology and molecular docking technique with the intention of providing certain foundation for future research on the clinical application and in-depth development of Lycii Cortex.

\section{Materials and Methods}

\subsection{Potential Active Components of Lycii Cortex}

The potential active components of Lycii Cortex were collected from TCMSP database ${ }^{[18]}$ (TCMSP, http://tcmspw.com/tcmsp.php) by setting the oral bioavailability $(\mathrm{OB}) \geq 30 \%$ and the drug-likeness $(\mathrm{DL}) \geq$ 0.18 and from BATMAN-TCM database ${ }^{[19]}$ (http://bionet.ncpsb.org/batman - tcm/) by setting a score cutoff $>20$ and $P<0.05$.

\subsection{Potential Targets in Treatment}

Targets related to the active components of Lycii Co rtex were identified by inputting the SMILES strings of active components, obtained from PubChem data base $^{[20]}$ (https://pubchem.ncbi. nlm.nih.gov/), into Swis sTargetPrediction $^{[21]}$ (http://www. Swisstargetprediction. ch /) for targets prediction. T2DM-related targets wer e screened out from human genome database GeneC $\operatorname{ards}^{[22]}$ (https://www.genecards.org/) with "Type 2 dia betes" and "T2DM" as key words.

The common targets gained by overlapping targets related to the active components of Lycii Cortex and T2DM targets using the online tool, Image GP (http://www.ehbio.com/Image GP/) were taken as the potential therapeutic targets of Lycii Cortex in the treatment of T2DM.

\subsection{Enrichment Analysis}

The obtained potential targets were imported into the database of DAVID ${ }^{[23]}$ (https://david.ncifcrf.gov/) for gene ontology (GO) enrichment analysis and Kyoto Encyclopedia of Genes and Genomes (KEGG) pathway analysis.

\subsection{Network Construction}

Cytoscape 3.6.1 ${ }^{[24]}$ was employed to build the "active components- targets" and the "interaction of potential targets" network diagrams, and network topology was utilized to analyze the degree of node, further constructing the "core components-targets" network.

\subsection{Molecular Docking}

The three-dimensional structures of Lycii Cortex's core components and effect targets were downloaded from Pubchem databases (https://pubchem.ncbi.nlm.nih.gov) and PDB databases (http://www.rcsb.org/). AutodockTool $4.0^{[25]}$ were used to add hydrogen and pre-treat target proteins. Molecular docking was carried out through Autodock vina $1.1 .2^{[26]}$ and the results were visualized by PyMOL ${ }^{[27]}$.

\section{Results}

\subsection{Construction of Active Components Base}

A total of 15 active components of Lycii Cortex were identified, shown in Table 1.

Table 1 Information table of effective components of Lycii Cortex

\begin{tabular}{lllll}
\hline TCMSP ID & Compound & OB $(\%)$ & DL & CAS Number \\
\hline MOL001552 & Hyoscyamine & 45.97 & 0.19 & $101-31-5$ \\
MOL001645 & Linoleyl acetate & 42.10 & 0.20 & $5999-95-1$ \\
MOL001689 & acacetin & 34.97 & 0.24 & $80-44-4$ \\
MOL002218 & scopolin & 56.45 & 0.39 & $531-44-2$ \\
MOL002219 & Atropine & 34.53 & 0.21 & $51-55-8$ \\
MOL002222 & sugiol & 36.11 & 0.28 & $511-05-7$ \\
MOL002224 & aurantiamide acetate & 58.38 & 0.59 & $56121-42-7$ \\
MOL000296 & hederagenin & 36.91 & 0.75 & $465-99-6$ \\
MOL000358 & beta-sitosterol & 36.91 & 0.75 & $83-46-5$ \\
MOL000449 & Stigmasterol & 43.83 & 0.76 & $83-48-7$ \\
MOL000953 & Cholesterol & 37.87 & 0.68 & $57-88-5$ \\
MOL002228 & Kulactone & 45.44 & 0.82 & $22611-36-5$ \\
MOL001790 & Linarin & 39.84 & 0.71 & $480-36-4$ \\
MOL000430 & Betaine & 40.92 & 0.01 & $107-43-7$ \\
MOL002221 & Kukoamine A & 1.42 & 0.56 & $75288-96-9$ \\
\hline
\end{tabular}




\subsection{Construction of Potential Targets Base}

The 3D structures of Lycii Cortex components were uploaded to the website of SwissTargetPrediction for target prediction of compounds, and the results showed 597 relevant targets after removing duplicates.

In the database of GeneCards, the search result with "Type 2 diabetes" as the key word revealed 12,272 related targets and that with "T2DM" as key word delivered 1,177 related targets. After the intersection, 1,176 common targets were identified, which were regarded as T2DM targets.

The final potential targets were the common targets (119) after intersecting Lycii Cortex components-related targets and T2DM targets, and the interaction network of common targets was drawn. Finally, a total of 12 core targets were selected from the network after setting a value of degree $>2$ times, as displayed in Table 2. And the analysis manifested that the following targets had the highest value of degree: glyceraldehyde-3-phosphate dehydrogenase (GAPDH), interleukin 6 (IL6), tumor necrosis factor (TNF), mitogen-activated protein kinase 3 (MAPK3), and cysteine protease 3 (CASP3), etc.

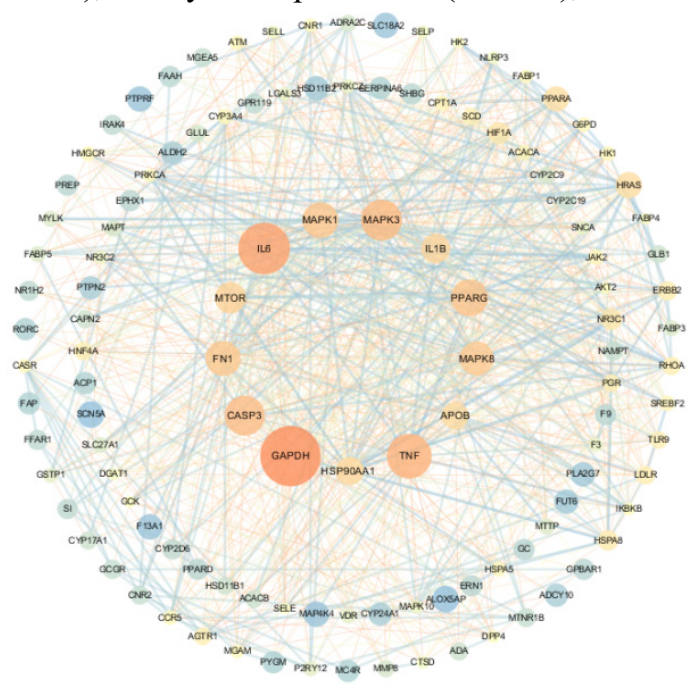

Fig.1 Common targets for disease of T2DM and Lycii Cortex treatment

Table 2 Core targets information table of Lycii Cortex in the treatment of T2DM

\begin{tabular}{|c|c|c|c|c|c|}
\hline No. & PDB ID & Gene & Protein & Degree & $\begin{array}{c}\text { Betweenness } \\
\text { Centrality }\end{array}$ \\
\hline 1 & 6IQ6 & GAPDH & $\begin{array}{c}\text { Glyceraldehyde-3-phosphate } \\
\text { dehydrogenase }\end{array}$ & 66 & 0.15 \\
\hline 2 & 4QIK & IL6 & Interleukin 6 & 57 & 0.09 \\
\hline 3 & 1FT4 & TNF & Tumor Necrosis Factor & 50 & 0.08 \\
\hline 4 & $2 \mathrm{ZOQ}$ & MAPK3 & Mitogen-activated protein kinase 3 & 46 & 0.04 \\
\hline 5 & 2XYP & CASP3 & Caspase- 3 & 45 & 0.03 \\
\hline 6 & $1 \mathrm{ZGY}$ & $\operatorname{PPAR} \gamma$ & $\begin{array}{l}\text { Peroxisome proliferator-activated } \\
\text { receptor } \gamma\end{array}$ & 42 & 0.07 \\
\hline 7 & $3 \mathrm{~W} 55$ & MAPK1 & Mitogen-activated protein kinase 1 & 41 & 0.04 \\
\hline 8 & 3M7P & FN1 & Fibronectin & 40 & 0.05 \\
\hline 9 & 3PZE & MAPK8 & Mitogen-activated protein kinase 8 & 39 & 0.02 \\
\hline 10 & 1FAP & MTOR & $\begin{array}{c}\text { Serine/threonine-protein kinase } \\
\text { mTOR }\end{array}$ & 36 & 0.01 \\
\hline 11 & 3LTQ & IL1 $\beta$ & Interleukin-1 $\beta$ & 34 & 0.02 \\
\hline 12 & 4BQG & HSP90AA1 & Heat shock protein HSP $90-\alpha$ & 33 & 0.02 \\
\hline
\end{tabular}

\subsection{Enrichment Analysis}

12 core targets were put into the DAVID database for GO enrichment analysis and KEGG pathway analysis, eventually identifying $50 \mathrm{GO}$ terms. The first $20 \mathrm{GO}$ biological process terms (Figure 2) and 20 KEGG pathways (Figure 3) were selected from the analysis results of core targets such as GAPDH, IL6, TNF, MAPK3, and CASP3.

It was shown that the core targets were able to regulate molecular functions like cytokine activity, affect cytoplasm, microtubule cytoskeleton, exosome, and other cellular components, and involved in a variety of biological processes like the phosphorylation of peptidylthreonine, lipopolysaccharide (LPS)-mediated signaling pathway, the positive regulation of cyclase activity, the positive regulation of genetic expression, and lipoprotein translocation. The results indicate that the above biological processes might be connected with the treatment of T2DM by Lycii Cortex.

KEGG pathway analysis delivered 76 pathways and Figure 3 exhibited the 20 most significant metabolic pathways together with their corresponding primary pathways and the acting core targets. As illustrated in Figure 3, the core targets were mainly distributed in the T2DM signaling pathway, PI3K-Akt signaling pathway, TNF signaling pathway, HIF-1 signaling pathway, MAPK signaling pathway, Toll-like receptor signaling pathway, and NOD-like receptor signaling pathway.

These results suggest that the active components of Lycii Cortex probably play a role in the treatment of $\mathrm{T} 2 \mathrm{DM}$ via these metabolic pathways. 


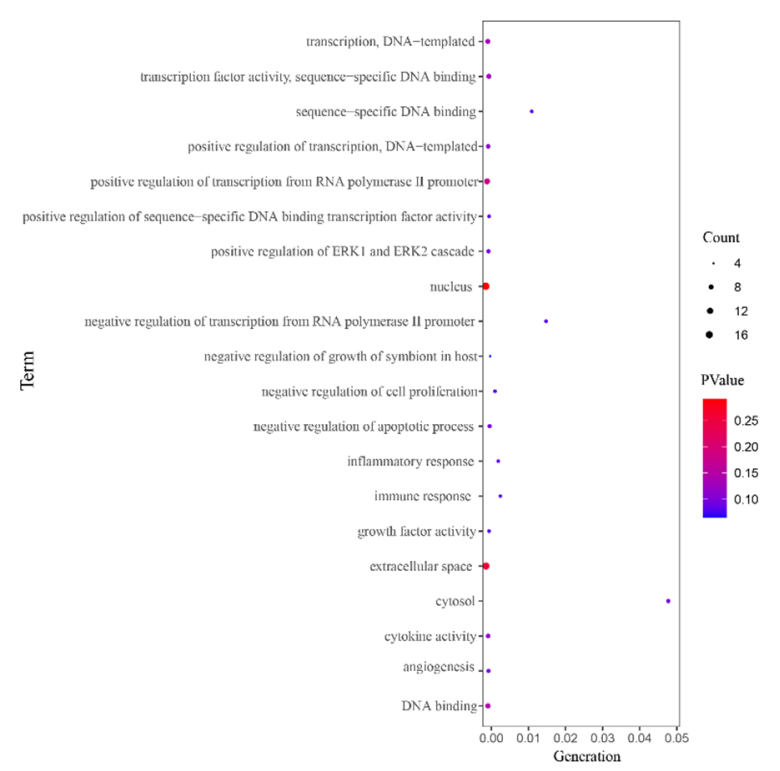

Fig. 2 GO enrichment analysis of Lycii Cortex in treating T2DM

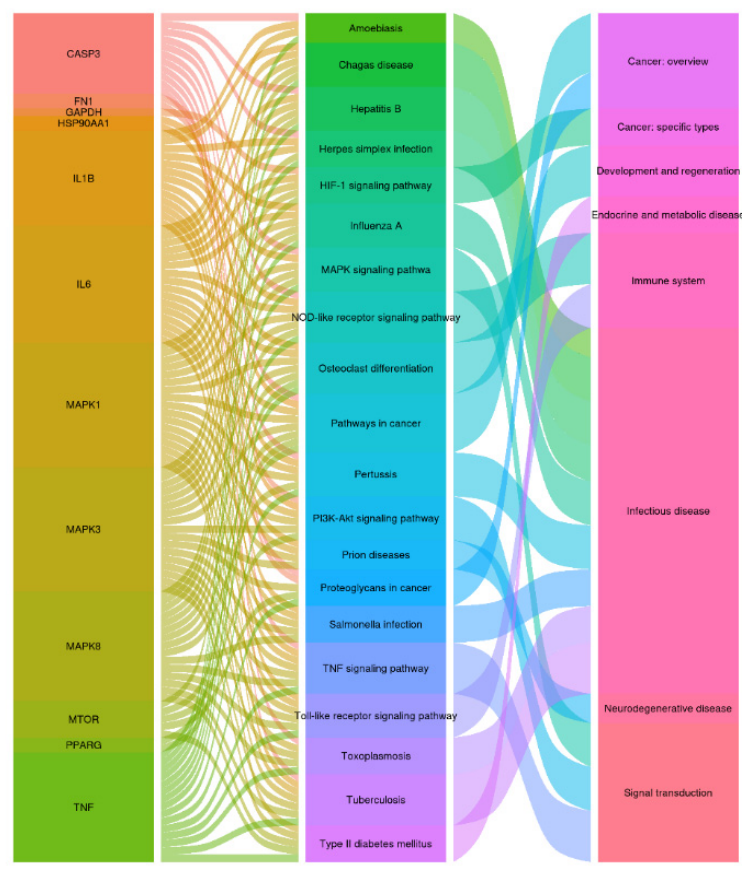

Fig. 3 KEGG pathway analysis of Lycii Cortex in treating T2DM

\subsection{Network Construction and Analysis}

\subsection{1 "Active components-Targets" Network}

The "active components-targets" network was built by importing these 15 active components and 597 effect targets into Cytoscap3.6.1. As exhibited in Figure 4, the red nodes stood for 15 active components and the purple ones for 597 effect targets with a network centrality of 0.158 and an average number of neighbor nodes of 1.951 . Each line in the network meant the potential interactive relationship between active components and effect targets.
It can be noticed that a single component may connect with more than one target, and different components also could link to the same target, indicating that the multicomponent and multi-targeted collaborative treatment of T2DM by Lycii Cortex.

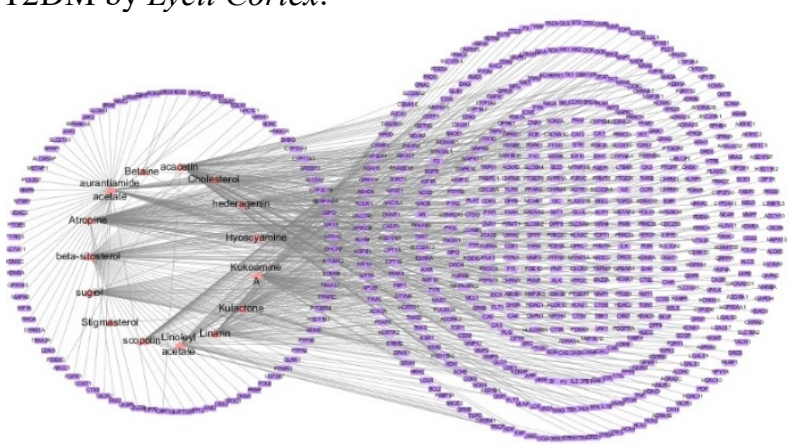

Fig.4 Network diagram of active components - targets in Lycii Cortex

\subsection{2 "Core components-Targets" Network}

The union set of "active components-targets" network and potential targets was obtained through the Merge function of Cytoscape 3.6.1 so as to derive the "core componentstargets" network in the treatment of T2DM by Lycii Cortex. The following six components were identified as the core components: kulactone, linarin, hederagenin, scopolin, etc.

\subsection{3 "Core components-Targets-Pathways" Network}

The "core components-targets-pathways" network was gained through the Merge function of Cytoscape3.6.1 as displayed in Figure 5 where the blue nodes represented core components, the purple ones meant effect targets, and the red ones stood for the pathways. It can be found that targets like GAPDH, IL6, TNF, MAPK, and CASP3 were primarily linked to the PI3K-Akt signaling pathway, TNF signaling pathway, MAPK signaling pathway, and HIF-1 signaling pathway.

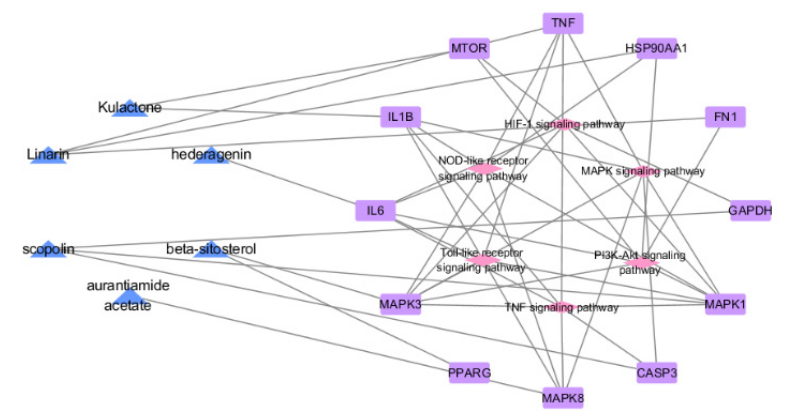

Fig.5 Core components-targets-pathways network

\subsection{Molecular Docking Analysis}

In this paper, the prediction results of network pharmacology were verified through the molecular docking. The docking analysis between core components and targets of Lycii Cortex in treating T2DM was performed based on the "core components-targets" 
network with metformin, which has a therapeutic effect in treating T2DM, as the positive control. The binding results were shown in Table 3 (no matched crystal structure for acetic acid ester) and the docking conformation of those with the lowest binding energy was visually analyzed using Pymol. As detailedly displayed in Figure 6, it is generally believed that a lower binding energy indicates a higher possibility of interaction between ligands and receptors and a higher number of hydrogen bonds denotes a more stable binding conformation between ligands and receptors. The binding force between these five ingredients and target proteins were considered strong when compared with the binding results of metformin in the control group. Figure 6-A exhibited the hydrophobic interaction between hederagenin and mTOR where one hydrogen bond was formed with TYR-26 as its amino acid residue at a distance of 2.2; Figure 6-E unveiled the hydrophobic interaction between kulactone and IL6 where 5 hydrogen bonds were formed with THR-167, ARG-164, U-9, C-10, and G-14 as their amino acid residues separately at a distance of $2.1,2.5,2.7,2.3$, and 2.2, which illustrated the strong binding force between these ingredients and core targets. The above results have demonstrated a strong binding force between core components of Lycii Cortex and T2DM targets, revealing an outstanding efficacy of Lycii Cortex in treating T2DM.

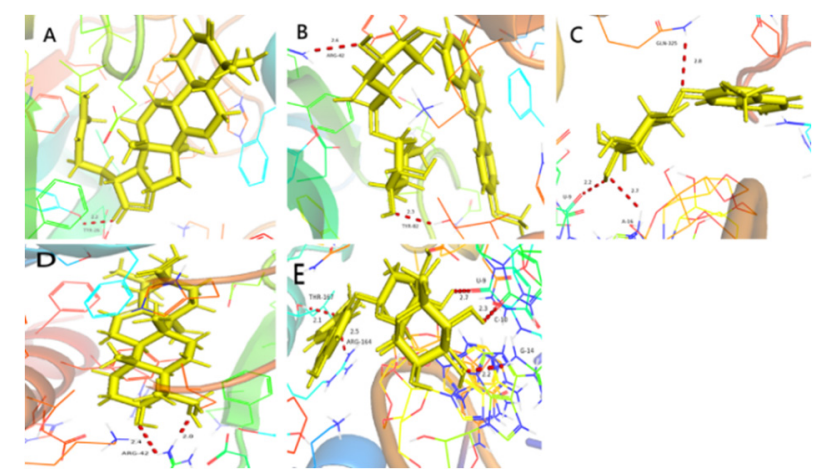

Fig.6 Docking schematic diagram of core components and targets (A: hederagenin-mTOR, B: Kulactone-mTOR, C: Linarin-IL6, D: $\beta$-sitosterol-mTOR, E: Kulactone-IL6)

Table 3 Binding results of ingredients of Lycii Cortex with targets (kcal/mol)

\begin{tabular}{ccccccc}
\hline Target & Scopolin & Hederagenin & Linarin & Kulactone & $\beta$-Sitosterol & Metformin \\
\hline GAPDH & -6.2 & -5.0 & -6.2 & -5.2 & -5.2 & -4.0 \\
IL6 & -8.4 & -7.8 & -8.4 & -10.7 & -7.3 & -4.9 \\
TNF & -5.4 & -5.4 & -5.4 & -6.2 & -5.5 & -3.5 \\
MAPK3 & -6.9 & -6.5 & -6.9 & -7.7 & -6.3 & -4.7 \\
CASP3 & -6.8 & -6.6 & -6.8 & -6.8 & -5.2 & -4.2 \\
PPAR $\gamma$ & -7.5 & -5.8 & -7.5 & -9.5 & -2.9 & -5.0 \\
MAPK1 & -7.9 & -8.1 & -7.9 & -9.2 & -7.3 & -4.4 \\
FN1 & -7.4 & -7.5 & -7.4 & -9.4 & -7.0 & -4.9 \\
MAPK8 & -7.8 & -8.2 & -7.8 & -8.3 & -4.3 & -3.9 \\
mTOR & -7.8 & -9.3 & -7.8 & -9.8 & -9.2 & -4.0 \\
IL1B & -5.8 & -6.5 & -5.8 & -6.3 & -6.1 & -4.2 \\
HSP90AA1 & -8.9 & -1.1 & -8.9 & -8.3 & 1.2 & -4.5 \\
\hline
\end{tabular}

\section{Discussion}

Previous researches determined an encouraging efficacy of Lycii Cortex in the treatment of T2DM, yet the underlying mechanism still remains unclarified ${ }^{[28,29]}$. In this study, network pharmacology and molecular docking technology were adopted to investigate the multicomponent, multi-targeted, and multi-pathway network of Lycii Cortex in treating T2DM. The results of the "core components-targets-pathway" network demonstrated that ingredients of Lycii Cortex such as kulactone, linarin, and hederagenin probably played a role in the treatment of T2DM through regulating T2DM signaling pathway, PI3K-Akt signaling pathway, TNF signaling pathway, HIF-1 signaling pathway, MAPK signaling pathway, etc., via core targets like GAPDH, IL6, TNF, and MAPK3

\subsection{Active Components of Lycii Cortex in Treating T2DM}

This study totally identified 15 active components of Lycii Cortex from databases and different components might play a collaborative role in the treatment of T2DM. The "core components-target" network diagram manifested five core components, including kulactone, $\beta$-sitosterol, linarin, hederagenin, and scopolin. Other report illustrated that linarin was capable of fulfilling a long-term, stable reduction of blood pressure among spontaneously hypertensive rats $(\mathrm{SHR})^{[30]}$ and attaining in vitro inhibition of LPS and TNF- $\alpha$-induced inflammatory injury in vascular endothelial cells. The anti-inflammatory action of $\beta$-sitosterol was possibly achieved by reducing the synthesis of NO, suppressing IL-6 activity of macrophage, and lowering the secretion of inflammatory factors such as IL-1 and TNF- $\alpha^{[31]}$. Meanwhile, hederagenin was endowed with a set of pharmacologic actions, for instance, antidiabetic and antilipemic capacities ${ }^{[32]}$. Q. C. Zhao et 
$a l^{[33]}$ found hederagenin could decrease the level of blood sugar and glucagon among normal mice, stimulate the secretion of insulin, and increase the level of hepatic glycogen and muscle glycogen. Moreover, hederagenin could play an anti-inflammatory role by inhibiting bradykinin or other inflammatory mediators ${ }^{[34]}$. Scopolin was proven to have the capacity of suppressing the metabolism of arachidonic acid as well as the ion carrierstimulated release of docosahexaenoic acid in mice peritoneal macrophage, and in vitro experiment also manifested its noticeable anti-inflammatory activity ${ }^{[35,36]}$. In sum, the core components identified in the "core components-targets" network diagram might be the potential active components of Lycii Cortex in treating T2DM.

\subsection{Core Targets of Lycii Cortex in Treating T2DM}

This study pinpointed 12 potential targets, encompassing GAPDH, IL6, TNF, MAPK3, CASP3, PPAR $\gamma$, as the core targets of Lycii Cortex in the treatment of T2DM, and the binding results illustrated a strong binding force between IL-6, FN1, PPAR $\gamma$, mTOR, MAPK3, MAPK8 and their corresponding active components.

IL-6 served as a multifunctional cytokine with the capacity of down-regulating the glucose transporter (GLUT-4) and insulin receptor substrate (IRS-1) and inhibiting the transportation of glucose into cells, thus affecting the transduction of insulin signals and triggering insulin resistance ${ }^{[37]}$. Besides, IL-6 could also elevate the level of free fatty acid, promote lipid peroxidation and enhance insulin resistance ${ }^{[38]}$. PPAR $\gamma$ was found to suppress inflammatory response via JAK, STAT, activator protein-1 (AP-1), NF-rd3, and nuclear factor of activated $\mathrm{T}$ cell (NFAT) signaling pathways ${ }^{[39]}$. MAPK3 and MAPK8 had a critical part in external factor-stimulated cellular reaction cascades ${ }^{[40]}$. The phosphorylation of MAPK8 contributed to the activation of the transcription factor, AP-1, further initiating the expression of a series of downstream genes and playing an important regulatory role in inflammation and other pathological processes ${ }^{[41]}$. mTOR was recognized as a negative regulatory factor of autophagy and its binding with an mTOR-specific inhibitor could suppress the protein kinase activity of mTOR and resist the effect of high glucose by enhancing autophagy ${ }^{[42]}$. The verification by molecular docking displayed a high binding activity between core components of Lycii Cortex and their corresponding effect targets, hinting that those targets might be essential targets in the treatment of T2DM by Lycii Cortex.

\subsection{Mechanism of Lycii Cortex in Treating T2DM}

GO enrichment analysis results manifested the involved cellular processes predominantly included the positive regulation of transcription from RNA polymerase II promoter, peptidyl-threonine phosphorylation, the reaction of exogenous dsRNA, the positive regulation of genetic expression, and the negative regulation of apoptosis process, the concerned cellular components contained cytoplasm, exosome, extracellular space, and microtubule cytoskeleton, and the associated molecular functions mainly encompassed ATP binding, cytokine activity, and protein binding. It implies that the likely biological processes involved in the treatment of T2DM by Lycii Cortex contain exogenous metabolism, redox reaction, control of cell apoptosis, genetic expression, and so on.

In addition, KEGG pathway analysis exhibited significant enrichment in pathways like TNF signaling pathway, PI3K-Akt signaling pathway, Toll-like receptor signaling pathway, and NOD-like receptor signaling pathway with the highest number of linked targets, and their involvement in the treatment of T2DM was accompanied with other pathways, which might explain the complex pathogenesis of T2DM. In the PI3K-Akt signaling pathway, the activated AKT could boost the transport of glucose into cells, mediate the intake of glucose, glycogen synthesis and degradation, thus lowering the level of blood glucose ${ }^{[43]}$. The activation of MAPK signal cascades was able to suppress the signal transduction of MAPK and down-regulate the expression of GLUT4, leading to the decreased transport of glucose $^{[44]}$. The phosphorylation of IRS resulted in insulin resistance ${ }^{[45]}$. In the TNF signaling pathway, the interaction of TNF $\alpha$ and $N F-\kappa B$ constituted the network of an inflammatory response, and their collaboration and circulation could deliver a pro-inflammatory effect ${ }^{[46]}$. HIF was identified as a kind of transcription factor activated under ischemic and anaerobic conditions, which could facilitate the treatment of T2DM by participating in the transcription regulation of angiogenesis, glucose metabolism, oxygen transport and modulating a number of downstream target genes ${ }^{[47]}$.

Based on the above-mentioned researches, it can be held that Lycii Cortex plays a role in the treatment of T2DM primarily by regulating the glycometabolism through the PI3K-Akt signaling pathway, controlling cell apoptosis via the TNF and MAPK signaling pathways, further declining the expression of inflammatory factors like TNF- $\alpha$, IL-6, and IL- $1 \beta$ in the HIF-1 signaling pathway. And the potential molecular mechanism was described in Figure 7.

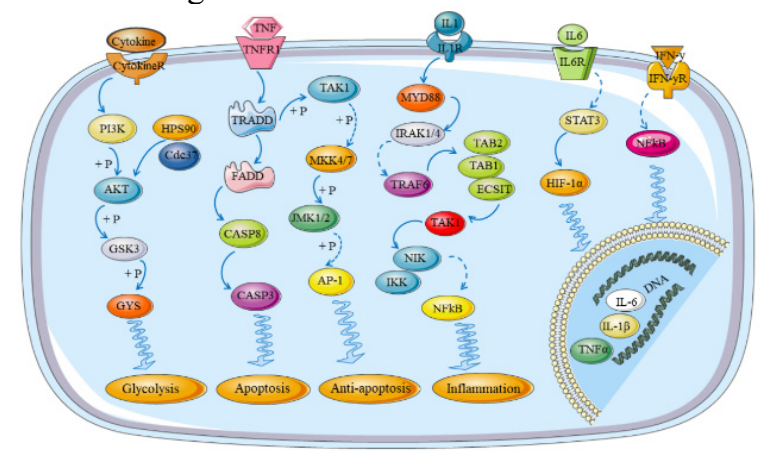

Fig. 7 Molecular mechanism of Lycii Cortex in treating T2DM (+p-phosphorylation)

In conclusion, network pharmacology and molecular docking were adopted in this study to analyze the potential active components and possible molecular mechanisms of Lycii Cortex in treating T2DM. And the results showed 
that the therapeutic effect of Lycii Cortex in the treatment of T2DM may be mainly through its active components like kulactone, linarin, hederagenin, and scopolin modulating signaling pathways like PI3K-Akt, TNF, HIF1, MAPK, and Toll-like receptor pathways via IL-6, FN1, PPAR $\gamma$, mTOR and other targets, eventually affecting biological processes such as peptidyl-threonine phosphorylation, LPS-mediated signaling pathway, the positive regulation of genetic expression, and the positive regulation of transcription from RNA polymerase II promoter.

\section{Reference}

1. S. V. Arnold, D. L. Bhatt, G. W. Barsness, Circulation, 141(19), 779-806 (2020).

2. A. K. Sikalidis, A. Maykish, Biomedicines, 8(1), 8 (2020).

3. P. Saeedi, I. Petersohn, P. Salpea, Diabetes Res Clin Pr, 157: 107843 (2019).

4. C. J. Bailey, A. A. Tahrani, A. H. Barnett, Lancet Diabetes Endo, 4(4), 350-359 (2016).

5. C. T. Chao, J. Wang, J. W. Huang, Front Pharmacol, 9, 860 (2018).

6. Y. Zhang, H. An, S. Y. Pan, Evid-Based Compl Alt, 111 (2016).

7. Q. H. Wu, B. T. Li, J. Tu, Chin Pharm J, 44(6), 11041109 (2019).

8. The Pharmacopeia of the People's Republic of China, Part I: The Pharmacopoeia Commission of PRC China Medical Science Press, (2020).

9. D. Qian, Y. X. Zhao, G. Yang, Molecules, 22(6), 911 (2017).

10. X. Yao, Y. Peng, L. J. Xu, Chem Biodivers, (2011).

11. D. Gao, Q. Li, Z. Liu, Yakugaku Zasshi, 127(10), 1715 (2017).

12. Z. Ye, Q. Huang, H. X. Ni, Phytotherapy Research Ptr, 22(12), 1665-1670 (2010).

13. Y. W. Chan, F. C. Lam, P. C. Leung, Phytotherapy Research, 23(5), 658-665 (2010).

14. X. M. Wu, C. F. Wu, Chin J Nat Medicines, 13(001), $1-2(2015)$

15. W. Hu, W. J. Fu, X. Wei, Evid Based Complement Alternat Med, 5276865 (2019).

16. L. Lai, J. Pei, Y. Yuan, Curr Pharm Des, 19(12), 232633 (2013).

17. X. Song, Y. Zhang, E. Dai, Int immunopharmacology, 80, 106179 (2014).

18. A. P. Davis, C. J. Grondin, R. J. Johnson, 47(D1), D948-D954 (2019).

19. Z. Liu, F. Guo, Y. Wang, Sci Rep, 6, 21146 (2016).

20. Q. Li, T. Cheng, Y. Wang, Drug Discov Today, 15(23/24), 1052-1057 (2010).

21. V. Zoete, A. Daina, C. Bovigny, J Chem Inf Model, 56(8), 1399-1404 (2016).
22. S. Marilyn, S. Irina, S. Orit, Bioinformatics, 18(11), 1542-1543 (2002).

23. C. T. Lopes, F. Max, K. Farzana, Bioinformatics, 26(18), 2347-2348 (2010).

24. D. W. Huang, B. T. Sherman, R. A. Lempicki, Nat Protoc, 4(1), 44-57 (2009).

25. G. M. Morris, R. Huey, W. Lindstorm, J Comput Chem, 30(16), 2785-2791(2009).

26. O. Trott, A. J. Olson, J Comput Chem, 31(2), 455-461 (2010).

27. M. A. Lill, M. L. Danielson, J Comput Aided Mol Des, 25(1), 13-19 (2011).

28. S. Funayama, K. Yoshida, C. Konno, Tetrahedron Letters, 21(14), 1355-1356 (1980).

29. Y. W. Chan, P. C. Leung, C. T. Che, Phytotherapy Research, 22(2), 190-196 (2008).

30. Q. Yin, S. Chen, M. Su, Evid-Bsded Compl Alt, 2014: 394276 (2014).

31. Choi, N. Ji, Choi, Y. Hyeok, Lee, M. Jeong, Nat Prod Res, 26(24), 2340-2343 (2016).

32. K. E. Hye, B. Seungho, S. Daiha, Oxid Med Cell Longev, 5498908 (2017).

33. Q. C. Zhao, B.X. Wang, S. W. Chen, CN 1385158 A. 2002-12-18.

34. G. J. Kim, D. H. Song, H. S. Yoo，Nutrients，9(1), 41 (2017).

35. A. Gepdiremen, V. Mshvildadze, H. Süleyman, Phytomedicine, 12(6-7), 440-444 (2005).

36. M. X. Xie, Y. Zhou, K. Zou, J Chin Med Mater, 31(9), 1332-1334 (2008).

37. C. Li, C. Rui, W. Hua, Int J Endocrinol, 1-9 (2015).

38. K. Yamaguchi, T. Nishimura, H. Ishiba, Liver Int, 35(2), 550-61 (2015).

39. S. K. Dai, Usuda, Tsugiyasu, World J Cardiol, 2014(8), 744-754 (2014).

40. A. Kumar, U. K. Singh, S. G. Kini, Future Med Chem, 7(15), 2065-2086 (2015).

41. J. Hirosumi, G. Tuncman, L. F. Chang, Nature, 420(6913), 333-336 (2002).

42. L. Liu, L. Yang, B. Chang, Ren Fail, 40(1), 492-497 (2018).

43. X. Hu, S. Wang, J. Xu, Int J Mol Sci, 15(6), 1044610458 (2014) .

44. G. Mingrone, G. Rosa, P. D. Rocco, Int J Obes, 26(9), 1165-1172 (2002).

45. V. Aguirre, T. Uchida, L. Yenush, 275(12), 9047-54 (2000).

46. X. R. Yao, F. Xia, W. J. Tang, J South Med Univ, 37 (1), 56-62 (2017)

47. C. U. Vohwinkel, S. Hoegl, H. K. Eltzschig, J Appl Physiol, 119(10), 1157-1163 (2015). 